\title{
Intra-colony channel morphology in Escherichia coli biofilms is governed by nutrient availability and substrate stiffness
}

\author{
Beatrice Bottura ${ }^{* 1}$, Liam M. Rooney ${ }^{2,3}$, Paul A. Hoskisson ${ }^{2}$, Gail McConnell ${ }^{1}$ \\ ${ }^{1}$ Department of Physics, SUPA, University of Strathclyde, G4 0NG, Glasgow, UK \\ 2 Strathclyde Institute of Pharmacy and Biomedical Sciences, University of Strathclyde, G4 0RE, Glasgow, UK \\ ${ }^{3}$ Institute of Biological Chemistry, Biophysics and Bioengineering, Heriot-Watt University, EH14 4AS, Edinburgh, UK \\ * Corresponding author: beatrice.bottura@strath.ac.uk
}

\begin{abstract}
Nutrient-transporting channels are found throughout mature Escherichia coli biofilms, however the influence of environmental conditions on intra-colony channel formation is poorly understood. We report the effect of different substrate nutrient concentrations and agar stiffness on the structure and distribution of intra-colony channels in mature E. coli colony biofilms using fluorescence mesoscopy and quantitative image analysis. Intra-colony channel width was observed to increase non-linearly with radial distance from the centre of the biofilm and channels were, on average, $50 \%$ wider at the centre of carbon-limited biofilms compared to nitrogen-limited biofilms. Channel density also differed in colonies grown on rich and minimal medium substrates, with the former creating a network of tightly packed channels and the latter leading to well-separated, wider channels with easily identifiable edges. We conclude that intra-colony channel morphology in E. coli biofilms is influenced by both substrate composition and nutrient availability.
\end{abstract}

\section{Introduction}

Biofilms are biological structures formed by microorganisms in a variety of environments, and they consist of microbial cells embedded in a self-secreted extracellular matrix [1]. Biofilm-forming organisms have evolved numerous mechanisms to protect their constituent cells from external biological and physiochemical stresses, resulting in populations of cells that exhibit increased resistance to a wide range of deleterious agents when compared to planktonic cells [2].

Interactions between bacterial aggregates and external substrates are crucial to biofilm formation and development, with surface properties affecting cell size [3], colony shape [4], growth rate [5, 6], expansion rate [7], motility [8] and wetting capability [9]. Biofilm-surface interactions can trigger the exchange of biomolecules between different species [10], and have been shown to influence pathogenicity through mechanical deformation of soft substrates and epithelial cell monolayers [11]. As a result, biofilms are a major burden to public health and industrial sectors [12], though the mechanisms of biofilm formation and metabolic behaviours have only recently been explored across spatial scales relevant to these settings [13]. It is therefore essential to gain a deeper understanding of the mechanisms that govern how biofilms form and grow, and how the growth environment influences their structure.

Bacterial growth rate and biomass formation depend on the type [14] and concentration [5] of nutrients available [15]. Altering the nutrient substrate upon which a biofilm grows can illicit profound changes in biofilm morphology, growth dynamics, and mechanical properties $[16,17,18,19]$. Changing the bulk carbon or nitrogen concentration of growth media can alter pattern formation within biofilms [4, 20, 21], and recent studies on single-species biofilms have identified cross-feeding mechanisms for acetate [22] and alanine [23]. Additionally, varying substrate stiffness has been linked to altered attachment [24, 25], motility [8], growth dynamics [26], and expansion [27] of biofilm constituent cells. Water and nutrient transport mechanisms have been found inside mature biofilms [28, 29, 30], yet how these may be altered by changing environmental conditions is currently unknown.

The complex channel networks recently reported by Rooney et al. [31] were found to facilitate nutrient transport throughout mature $E$. coli biofilms, however it is uncertain if the architecture of those intra-colony channels is influenced by the substrate composition. It was hypothesised that intra-colony 
channel morphology changes according to nutrient availability and stiffness of the growth substrate. This hypothesis was tested using the Mesolens [32], an optical mesoscope which enables sub-micron resolution across multi-millimeter-size live biofilms. A custom image analysis workflow was also designed to measure intra-colony channel width under different environmental conditions to establish the role of the growth substrate on E. coli intra-channel morphology.

\section{Results}

\section{Nutrient availability affects biofilm morphology at both the microscale and the mesoscale}

To test how biofilm morphology is affected by nutrient availability, specific carbon and nitrogen limiting conditions were determined for E. coli JM105-miniTn7-gfp (Supplementary Figure 1). Carbon and nitrogen were limited by controlling the nutrient concentrations of the growth media (glucose and ammonium chloride, respectively). The limiting carbon concentration was $15 \pm 5 \mathrm{mM}$ (C:N ratio of 1:1.25) and the limiting nitrogen concentration was $2.5 \pm 1.5 \mathrm{mM}$ (C:N ratio 27:1). These are broadly compatible with previous E. coli growth experiments in minimal medium [33].

We investigated the impact of nutrient concentration on biofilm morphology by measuring the global biofilm property of base area (Figure 1). Biofilms grown on carbon-limited substrates and those grown on nitrogen-limited substrates were similar in size, with average base areas measuring $1.374 \pm 0.118 \mathrm{~mm}^{2}$ ( $\mathrm{n}=16$ colonies) and $1.668 \pm 0.166 \mathrm{~mm}^{2}(\mathrm{n}=18$ colonies $)$ for carbon- and nitrogen-limited colonies respectively. As expected, biofilms grown on carbon-rich substrates were larger than those grown under carbon limitation $(\mathrm{p}=0.00088, \mathrm{t}$-value $=2.20,11$ degrees of freedom). Carbon excess also led to larger biofilms than nitrogen excess $(\mathrm{p}=0.00090$, $\mathrm{t}$-value $=2.20,11$ degrees of freedom $)$ : biofilms grown on 200 $\mathrm{mM}$ carbon substrates had an average base area of $3.738 \pm 0.510 \mathrm{~mm}^{2}$ ( $\mathrm{n}=11$ colonies), whereas those grown on $10 \mathrm{mM}$ nitrogen had an average base area of $1.370 \pm 0.129 \mathrm{~mm}^{2}(\mathrm{n}=11$ colonies). Errors given correspond to the standard error on the mean across each dataset. The variation in base area measurements is a direct consequence of colony size variation across the substrate, which is affected by the relative proximity of adjacent competing colonies on the surface as well as by differences in metabolic activity between individual biofilms [34]. Our results show that carbon is the limiting factor governing biofilm base area during growth on minimal medium substrates.

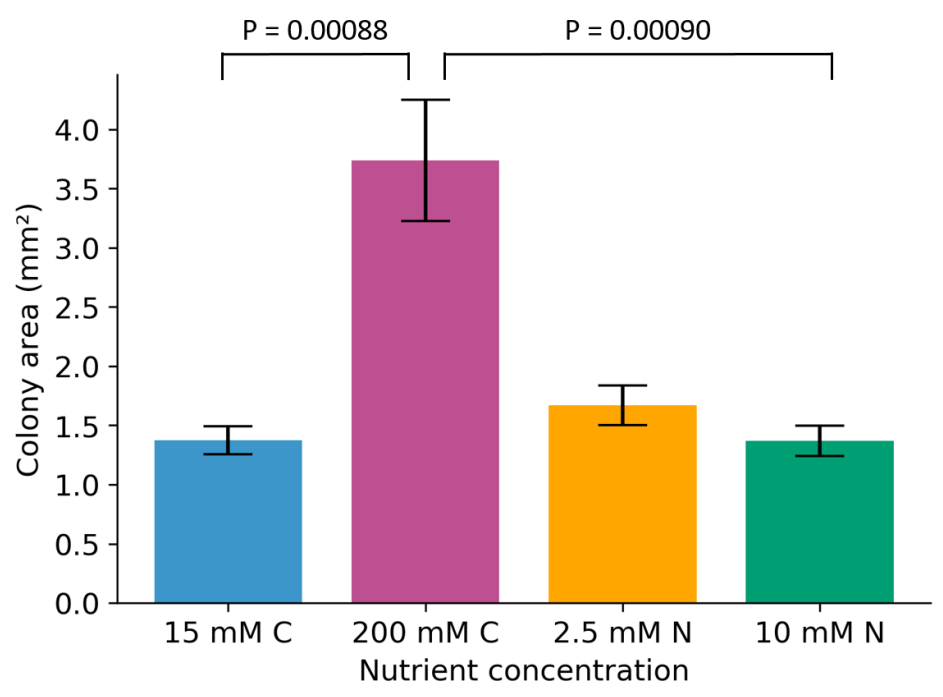

Figure 1: Base area of E. coli JM105-mini-Tn7-gfp biofilms calculated for four nutrient conditions. Error bars are standard errors of the mean across biological repeats ( $\mathrm{n} \geq 11$ for each condition). Two-tailed two-sample t-tests were performed on the data assuming unequal variances, with relevant $p$-values shown.

The maximum intensity projections (Figure 2) which are colour-coded by depth also indicate that not all biofilms have the uniform dome-shaped structure typical of E. coli biofilms [7, 35]. Instead, in Figure $2 \mathrm{c}$ we observe that the thickest region of the biofilm is located at an intermediate radius, between the centre and the edge of the colony. A phenomenon similar to colony sectoring is observed 
bioRxiv preprint doi: https://doi org/10.1101/2021.12.17.473116; this version posted December 18, 2021. The copyright holder for this preprint (which was not certified by peer review) is the author/funder, who has granted bioRxiv a license to display the preprint in perpetuity. It is made available under aCC-BY-NC-ND 4.0 International license.
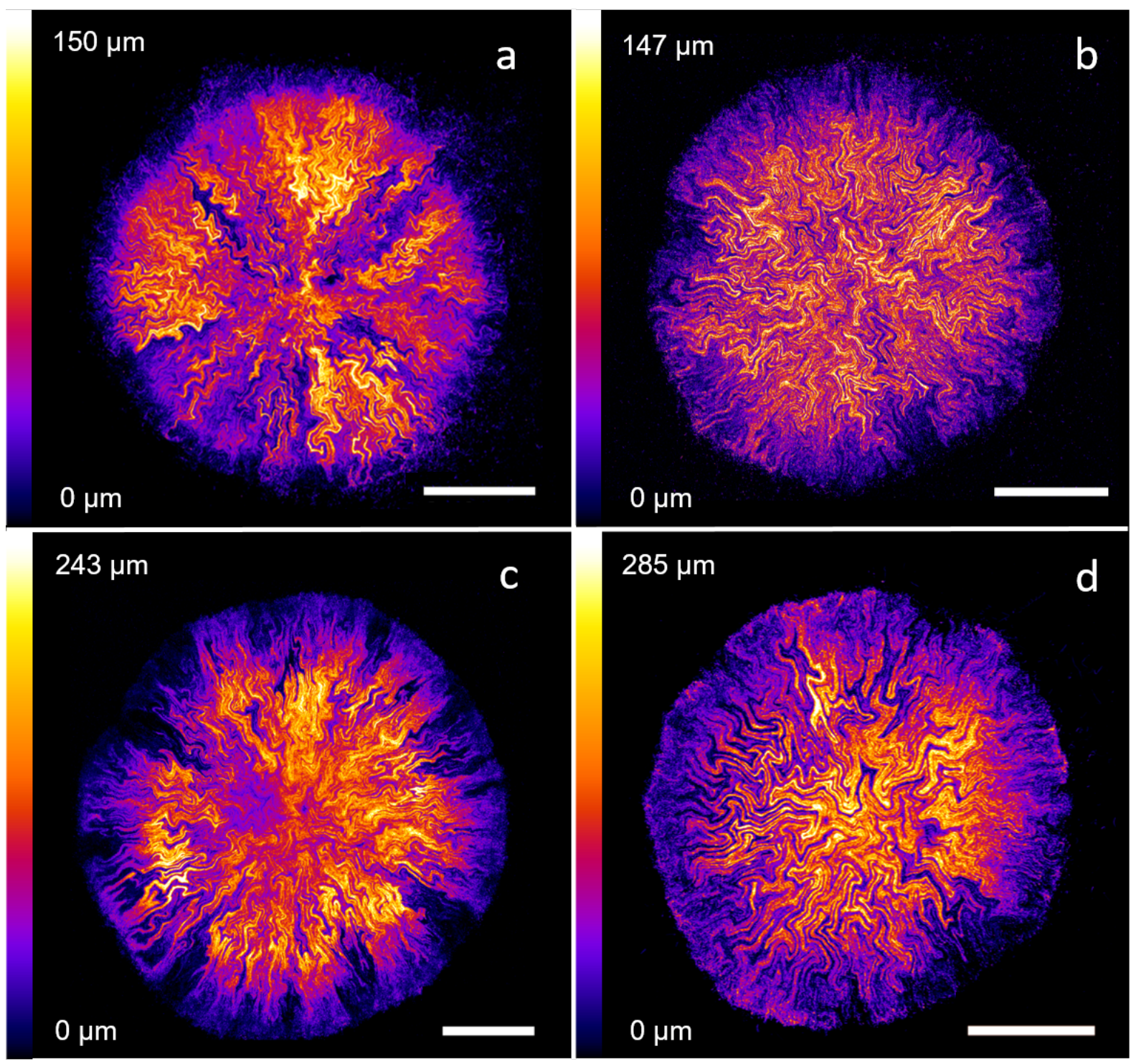

Figure 2: E. coli JM105-mini-Tn7-gfp colonies grown on M9 minimal medium substrates with 15 mM carbon (a), $200 \mathrm{mM}$ carbon (b), $2.5 \mathrm{mM}$ nitrogen (c) and $10 \mathrm{mM}$ nitrogen (d) concentrations. Carbon-limited substrates (a) lead to an average increase in colony base area of $38 \%$ with respect to nitrogen-limited substrates (c). Intra-colony channel patterns appear radially expanding from the centre on nutrient-limited substrates (a,c), whereas they have a fractal structure with sharp turns on nutrient-sufficient substrates $(b, d)$. The figure includes maximum intensity projections of confocal z-stacks made of images separated by $3 \mu \mathrm{m}$ in the z-direction. Images are colourcoded in the axial direction, with purple corresponding to the base of the biofilm and white corresponding to the top. Scale bars: $500 \mu \mathrm{m}$.

on carbon-limited substrates (Figure 2a), where radial sections of the colony have a considerably lower fluorescence signal than the rest of the colony. The pattern of intra-colony channels is also distributed heterogeneously inside the biofilm depending on the nutrient availability. Channels grown on nutrientlimited substrates appear to expand radially outwards from the centre in approximately straight lines, whereas on nutrient-rich substrates the channels often change direction sharply and do not follow a straight line. These observations confirm that the morphology of E. coli biofilms is strongly determined by nutrient availability.

\section{Intra-colony channel width increases non-linearly with radial distance from the centre of the colony}

Channel width was measured along whole circumferences in E. coli JM105-miniTn7-gfp biofilms grown on M9 minimal medium substrates with limitation and excess of both carbon and nitrogen. The average 
bioRxiv preprint doi: https://doi.org/10.1101/2021.12.17.473116; this version posted December 18, 2021. The copyright holder for this preprint (which was not certified by peer review) is the author/funder, who has granted bioRxiv a license to display the preprint in perpetuity. It is made available under aCC-BY-NC-ND 4.0 International license.

channel width was plotted against radial distance from the centre of the colony, revealing a non-linear increase in width with radius. Representative plots of $n=3$ repeats for each nutrient condition are shown in Figure 3. The minimum observed channel width was between $10 \mu \mathrm{m}$ and $15 \mu \mathrm{m}$, and corresponded to the innermost region of the biofilm (at $200 \mu \mathrm{m}$ radius). This value was not limited by the resolution of the image datasets, but rather by the smallest radial distance at which channel width could be accurately measured.

The modality of increase in intra-colony channel width was explored by applying exponential fits to each dataset. R-squared values of exponential fits were obtained across 3 replicates, and their averages were 0.778 for $15 \mathrm{mM}$ carbon, 0.664 for $200 \mathrm{mM}$ carbon, 0.805 for $2.5 \mathrm{mM}$ nitrogen and 0.870 for 10 $\mathrm{mM}$ nitrogen. By verifying that the increase in channel width along the colony radius is non-linear, we ensured that the change was not just a result of polar geometry.
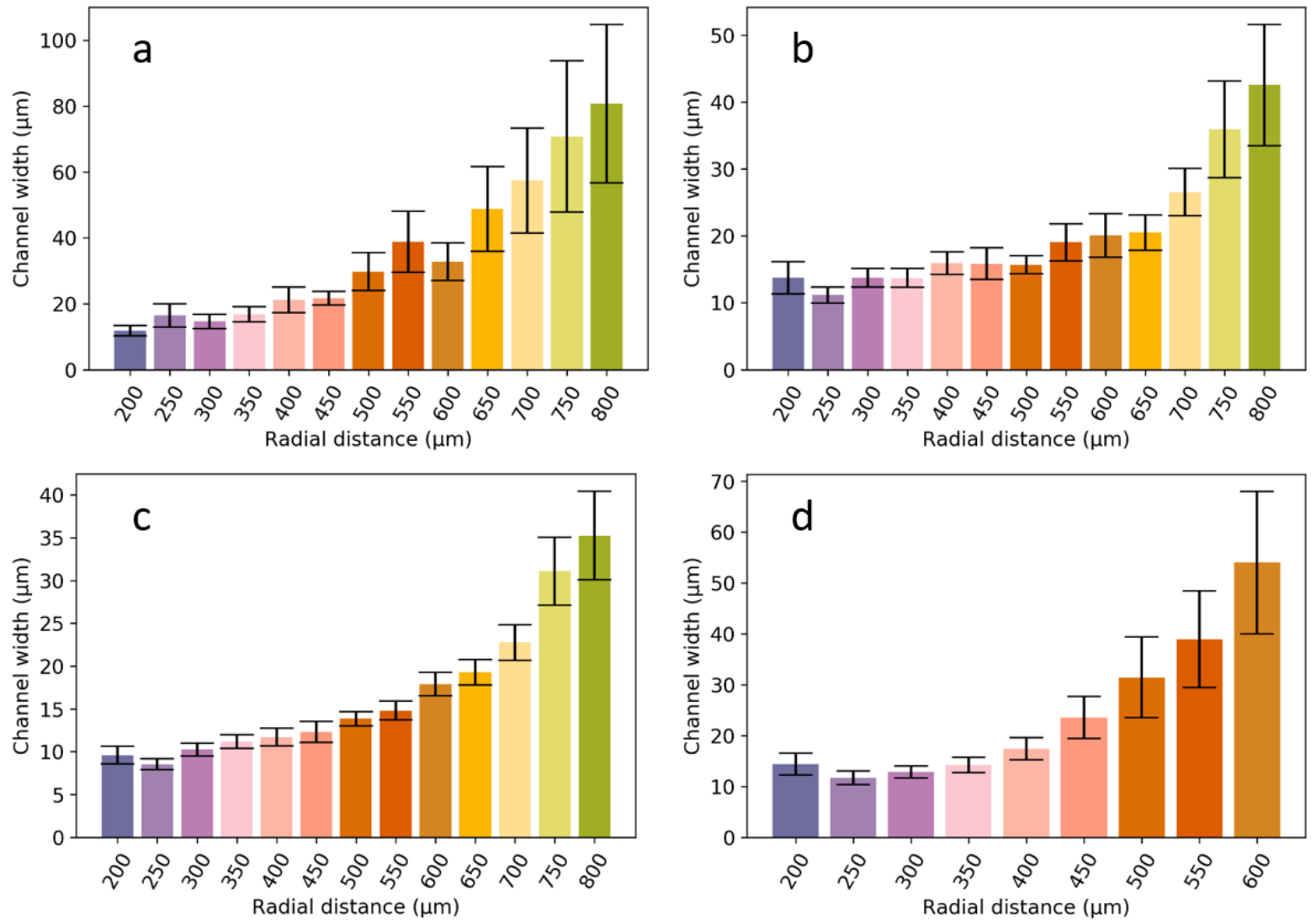

Figure 3: Non-linear channel width variation across the radial direction for E. coli JM105-miniTn7-gfp biofilms grown on M9 minimal medium substrates with $15 \mathrm{mM}$ carbon (a), $200 \mathrm{mM}$ carbon (b), $2.5 \mathrm{mM}$ nitrogen (c) and $10 \mathrm{mM}$ nitrogen $(\mathrm{d})$. Error bars represent the standard error on the mean of channel widths at each radial position. Each plot is representative of $n=3$ biological repeats.

\section{Carbon-limited colonies possess wider channels than nitrogen-limited colonies}

To investigate the effect of nutrient availability on intra-colony channel width, colonial biofilms were grown on substrates with a range of nutrient concentrations: $15 \mathrm{mM}$ carbon ( $\mathrm{n}=8$ colonies), 200 $\mathrm{mM}$ carbon ( $\mathrm{n}=7$ colonies), $2.5 \mathrm{mM}$ nitrogen $(\mathrm{n}=9$ colonies $)$ and $10 \mathrm{mM}$ nitrogen $(\mathrm{n}=4$ colonies $)$. Intra-colony channel width was compared across nutrient conditions at three normalised radial positions, revealing that channels were approximately $50 \%$ wider on carbon-limited substrates than on nitrogenlimited substrates inside the biofilm. This increase was most significant at the mid-radius region of each colony, where channel widths measured on average $21.25 \pm 1.26 \mu \mathrm{m}$ under carbon limitation and 14.38 $\pm 0.71 \mu \mathrm{m}$ under nitrogen limitation $\left(\mathrm{p}=2.49 \times 10^{-6}\right.$, t-value $=1.96,776$ degrees of freedom $)$.

These data suggest that carbon- and nitrogen-based nutrients mechanistically affect channel morphology in different manners. The number of channels detected inside the biofilm also varied differently depending on the nutrient source, and was largest at mid-radius. 


\section{Substrate stiffness affects colony size and channel density}

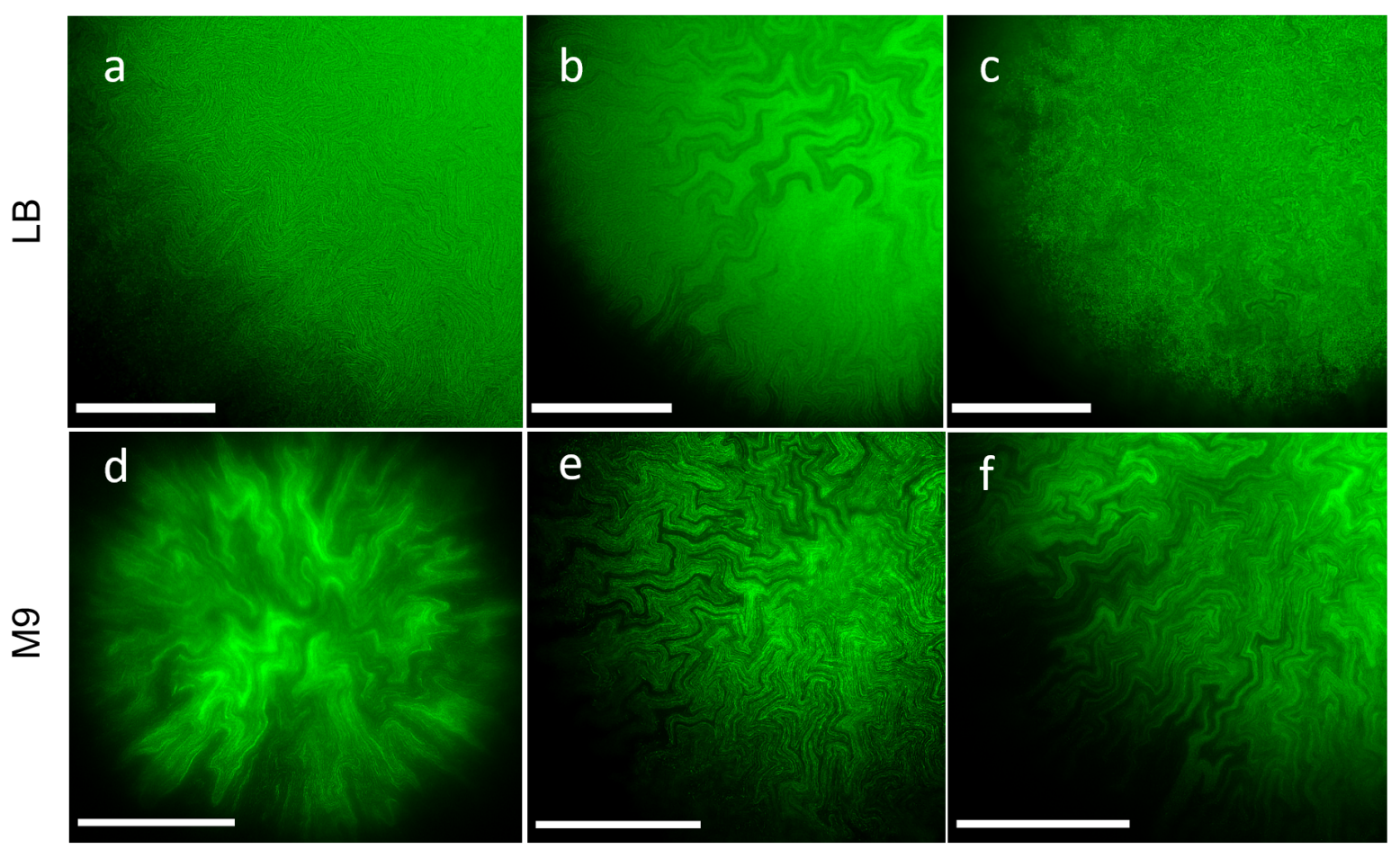

Figure 4: ROIs showing intra-colony channel morphologies of E. coli JM105-mini-Tn7-gfp colonies grown on solid LB (rich medium, top half) and solid M9 (minimal medium, bottom half). The agar concentrations of the substrates were $0.5 \%(\mathrm{a}, \mathrm{d}), 1 \%(\mathrm{~b}, \mathrm{e})$ and $2 \%(\mathrm{c}, \mathrm{f})$. All images were acquired using the Mesolens in widefield epi-fluorescence mode to capture the colonies at the midpoint along biofilm thickness. The images were acquired with M9 mounting medium and the Mesolens in water immersion, except for panel d, which was acquired in air immersion and no mounting liquid to prevent the colony from detaching from the substrate. All images were deconvolved using Huygens proprietary software. Scale bars: $500 \mu \mathrm{m}$.

The effect of substrate stiffness (agar concentration) on internal colony morphology was investigated by imaging biofilms grown on soft and hard agar substrates, in both rich and minimal medium (Figure 4). We observed an increase in out-of-focus fluorescence for biofilms grown on rich medium substrates compared to biofilms grown on minimal medium substrates. On minimal medium, channel borders were better resolved due to the higher contrast relative to the rest of the biofilm. On rich medium, the density of intra-colony channels increased with decreasing substrate stiffness. On $0.5 \%$ agar LB substrates (Figure 4a) channels were densely packed in the whole biofilm, while some widely-separated channels progressively appeared on portions of biofilms grown on stiffer substrates ( $1 \%$ and $2 \%$ agar concentration, Figures $4 \mathrm{~b}$ and $4 \mathrm{c}$ respectively).

Varying agar concentration was also associated with a change in biofilm base area for both rich and minimal medium substrates (Figure 5). On rich LB medium substrates, decreasing agar concentration resulted in an increase in biofilm base area, which measured on average $1.733 \pm 0.245 \mathrm{~mm}^{2}$ on $2 \%$ agar ( $\mathrm{n}=12$ colonies $), 3.080 \pm 0.477 \mathrm{~mm}^{2}$ on $1 \%$ agar $(\mathrm{n}=15$ colonies $)$ and $3.854 \pm 0.772 \mathrm{~mm}^{2}$ on $0.5 \%$ $\operatorname{agar}(\mathrm{n}=13$ colonies). Interestingly, the effect was opposite on M9 minimal medium substrates, where the average base area was $2.253 \pm 0.224$ on $2 \%$ agar $(\mathrm{n}=18$ colonies $), 2.434 \pm 0.184 \mathrm{~mm}^{2}$ on $1 \%$ agar ( $\mathrm{n}$ $=12$ colonies) and $1.387 \pm 0.142 \mathrm{~mm}^{2}$ on $0.5 \%$ agar $(\mathrm{n}=9$ colonies $)$. Two-tailed t-tests were performed between the $0.5 \%$ and the $2 \%$ agar concentration data for both rich and minimal medium, and revealed that agar stiffness had a greater effect on biofilm base area on minimal medium $(\mathrm{p}=0.0032$, $\mathrm{t}$-value $=$ 2.06, 25 degrees of freedom) than on rich medium $(\mathrm{p}=0.0202$, t-value $=2.14,14$ degrees of freedom $)$. The difference in base area between biofilms grown on rich and minimal medium substrates was most significant at $0.5 \%$ agar concentration $(\mathrm{p}=0.0078$, t-value $=2.16,13$ degrees of freedom $)$. 


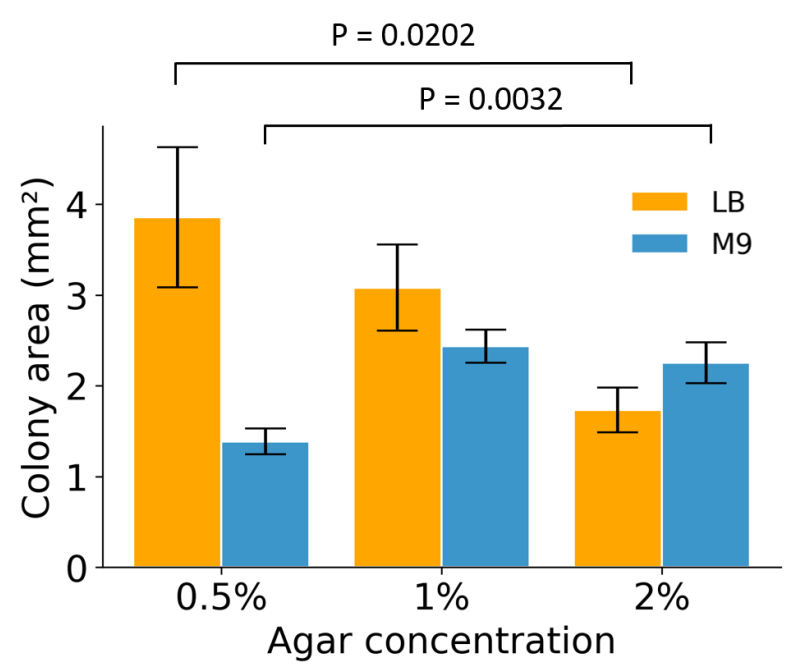

Figure 5: E. coli JM105-mini-Tn7-gfp biofilm base area calculated for three different agar concentrations of the substrate, in both rich (LB) and minimal (M9) medium. Error bars are standard errors on the mean across biological repeats ( $\mathrm{n} \geq 12$ for $\mathrm{LB}$ datasets and $\mathrm{n} \geq 9$ for $\mathrm{M} 9$ datasets). Two-tailed two-sample t-tests were performed on the data assuming unequal variances, with relevant p-values shown.

\section{Discussion}

This study constitutes the first quantitative analysis of environmental modulation of intra-colony channels in mature E. coli biofilms. Using a simple custom image analysis pipeline based on the open-source software FIJI and the Python programming language, it was possible to quantify biofilm properties at both the mesoscopic scale (by measuring biofilm base area) and at the microscale (by measuring intracolony channel width). Both quantities were used to investigate the effect of environmental conditions on biofilm morphology, which was found to be affected by both the type of substrate (rich or minimal medium agar) and by substrate properties (stiffness and nutrient concentration). Thanks to its simplicity, our pipeline can easily be adapted to the study of internal patterns of other biofilm-forming species. Our results show that intra-colony channel architecture is heavily affected by substrate composition.

The mesoscopic effects of nutrient limitation were studied by calculating biofilm substrate area. Nutrient abundance in the substrate, achieved by increasing the amounts of either glucose or ammonia, led to larger biofilms than nutrient limitation. Biofilm base area was most significantly affected by the carbon concentration in the growth substrate and was 2.7 times larger for biofilms grown on carbon excess than on carbon limitation. On the other hand, increasing the nitrogen concentration on a substrate with sufficient carbon availability did not significantly affect biofilm base area. These findings agree with the reported increase of biofilm formation with glucose concentration in Staphylococcus species [36] and the increase in colony size with glucose concentration for E. coli [37] and yeast [38].

Biofilm base area was also affected by substrate stiffness, but in opposite manners depending on the medium. An increase in agar stiffness in rich medium was associated with a reduction in colony size, due to slower radial expansion of biofilms on the surface brought by stronger frictional forces [7]. The trend of increased base area with decreased agar stiffness has previously been observed in Vibrio cholerae biofilms grown on LB medium with different agar concentrations [4]. The opposite trend was observed on minimal medium: biofilms grown on stiff substrates had a base area on average 1.6 times larger than those grown on semi-solid substrates. This could be due to faster assimilation of nutrients occurring on semi-solid minimal media with respect to stiff minimal media, causing a halt to radial expansion. Finally, colony size was on average almost 2.5 times larger on semi-solid rich medium substrates than on semi-solid minimal medium substrates. The slower growth of E. coli in minimal medium with respect to rich medium (with average doubling times of $18 \pm 0.5$ minutes in LB and $38 \pm 1.1$ minutes in M9glucose [39]) can explain this difference in biofilm base area only in part, since we observed no significant difference in base area between rich and minimal medium substrates at higher agar concentrations of $1 \%$ and $2 \%$.

Environmental conditions also altered the distribution of intra-colony channels inside biofilms. Channels formed on substrates with limiting carbon or nitrogen concentrations expanded radially outwards, in approximately straight lines. This could be because bacteria growing on nutrient-depleted substrates 
adhere less strongly on the surface of attachment, and expand more rapidly [40], or may be indicative of nutrient foraging behaviour. On the other hand, channels formed on nutrient-rich substrates formed complex fractal patterns. It is hypothesised that this type of structure emerges due to rapid cell growth and larger cell dimensions, thereby resulting in denser colonies creating a tighter network of channels. This in turn enables a greater proportion of cells to access the nutrients that are transported within the biofilm. A recent study by Fei et al. [4] highlighted two distinct types of patterns forming on the surface of $V$. cholerae biofilms, namely radial stripes and zigzag herringbone patterns. Conversely, the biofilms in our study only involved the same type of fractal patterns: intra-colony channel structures had homogeneous morphology, with only width and spacing varying depending on environmental conditions. Nonetheless, it is important to remember that the wrinkle patterns of $V$. cholerae and B. subtilis are substantially different from intra-colony channels in $E$. coli, since the former are surface features arising during biofilm expansion because of frictional forces, whereas the latter permeate the whole biofilm volume and have a complex 3D architecture and nutrient uptake function.

Furthermore, we found that substrate stiffness affected intra-colony channel density on rich medium substrates: channels were tightly packed and had a uniform, narrow width on semi-solid substrates, but as the agar concentration increased, wider channels appeared in portions of the biofilm. This observation appears to be in contrast with previous work on $V$. cholerae biofilms, where homogeneity in radial feature distribution and spacing at the edge of mature biofilms increased with agar stiffness [41]. An increase in out-of-focus fluorescence was also observed in widefield epi-fluorescence images of biofilms grown on rich medium compared to those grown on minimal medium. The fluorescence signal inside channels was likely due to bacterial cells being present inside them, as a result of movement inside the biofilm, collective reorientation [42] or shedding of cells from the inner walls of the channels. Previous studies on $V$. cholerae [43] confirmed that expansion in the depth direction is dependent on friction between the substrate and the expanding cells, with softer substrates leading to flatter biofilms than stiff substrates. This could explain the reduction in contrast between channel border and constituent cells observed on stiff rich medium substrates. A variation in internal biofilm architecture along the depth direction has also previously been reported for E. coli growing on agar substrates [44] and was attributed to differences in extracellular matrix components assembly and organisation inside the biofilm volume.

Finally, the variation in channel width inside mature biofilms was investigated: channel width increased non-linearly along the radial direction from the centre of the biofilm under all nutrient conditions. We hypothesise that channel width is larger at the edge of the colony due to rapid cell growth in nutrient sufficient conditions, resulting in non-uniform radial expansion, as previously observed for B. subtilis [40] and $V$. cholerae [4]. Remarkably, channels were on average $50 \%$ wider at the centre of carbon-limited media than inside nitrogen-limited media. Bacteria regulate their cell size and growth rate in response to nutrient availability [45], suggesting that the overall growth rate and cell dimensions affect the manner in which channels form across the colony, resulting in variations in channel dimensions as an emergent property of biofilm expansion.

Studying biofilm internal patterns at the cellular level is a task mostly relegated to theoretical modelling and computer simulations [7, 46, 47, 48, 49]. Experimental approaches in imaging biofilms at single-cell resolution $[50,51,52]$ have been limited in the total biofilm size that can be imaged at once (under $100 \mu \mathrm{m}^{2}$ ). This limitation has recently been overcome thanks to the Mesolens, which has enabled the discovery and characterisation of intra-colony channels inside $E$. coli biofilms and insight into how the environment shapes their formation.

\section{Methods}

\section{Materials and strains}

The non-pathogenic E. coli strain JM105 (endA1, recA1, gyrA96, thi, hsdR17 (rk-, mk+), relA1, supE44, $\Delta$ (lac-proAB), $\left[\mathrm{F}^{\prime}\right.$ traD36, proAB, laqIqZ $\left.\left.\Delta \mathrm{M} 15\right]\right)$ containing mini-T7 gfp [53], enabling GFP fluorescence, was used throughout the study. Liquid cultures were grown in Lysogeny Broth (LB) [54]. Minimal medium growth was in M9 medium [54], prepared in a 5x concentrated solution $\left(\mathrm{Na}_{2} \mathrm{HPO}_{4} 30\right.$ $\mathrm{g} / \mathrm{L}, \mathrm{KH}_{2} \mathrm{PO}_{4} 15 \mathrm{~g} / \mathrm{L}, \mathrm{NH}_{4} \mathrm{Cl} 5 \mathrm{~g} / \mathrm{L}, \mathrm{NaCl} 2.5 \mathrm{~g} / \mathrm{L}$ ) then diluted to $1 \mathrm{x}$ with distilled deionised water and supplemented with $1 \mathrm{mM} \mathrm{MgSO}_{4} \cdot 7 \mathrm{H}_{2} \mathrm{O}, 0.2 \%(\mathrm{w} / \mathrm{v})$ glucose and $0.00005 \%(\mathrm{w} / \mathrm{v})$ thiamine. Solid substrates were made by adding agar prior to autoclaving, in concentrations of $20 \mathrm{~g} / \mathrm{L}$ (LB agar) and $15 \mathrm{~g} / \mathrm{L}$ (M9 agar) for nutrient concentration experiments. For agar stiffness experiments, LB and M9 
media were prepared as described above, but the agar concentration was progressively reduced for soft substrates (giving $5 \mathrm{~g} / \mathrm{L}, 10 \mathrm{~g} / \mathrm{L}$ and $20 \mathrm{~g} / \mathrm{L}$ for both LB and M9 media).

\section{Imaging chamber design and 3D-printing}

The imaging chamber to be used with the Mesolens was designed on AutoCAD (Autodesk, USA) by modifying the original design of Rooney et al. [31]. The chamber was designed to mimic a Petri dish, and consisted of a $120 \mathrm{~mm}$ x $100 \mathrm{~mm}$ x $12 \mathrm{~mm}$ plate with a $60 \mathrm{~mm}$ diameter, $10 \mathrm{~mm}$ deep well at its centre to hold the agar substrate (Supplementary Figure 2). After 3D printing the chamber in black ABS plastic (FlashForge, Hong Kong) using a FlashForge Dreamer 3D printer (FlashForge, Hong Kong), the corners were smoothed out using a scalpel. This reduced the movement of the imaging chamber inside the square plate. The chamber was sterilised with a $70 \%(\mathrm{v} / \mathrm{v})$ ethanol solution, and then under UV light for 20 minutes immediately before the addition of sterile solid growth medium.

\section{E. coli biofilm specimen preparation}

Liquid cultures of E. coli JM105-mini-Tn7-gfp were prepared in LB medium supplemented with 25 $\mu \mathrm{g} / \mathrm{mL}$ gentamicin and incubated overnight at $37^{\circ} \mathrm{C}$. Overnight cultures were diluted (1:100) into fresh LB medium and incubated at $37^{\circ} \mathrm{C}$ until they reached an optical density of 0.5 (mid-exponential growth phase).

To grow biofilms on rich medium, a liquid culture with density $1 \times 10^{4}$ colony-forming units (CFUs) per $\mathrm{mL}$ was inoculated on the LB agar substrate inside the imaging chamber. The specimen was incubated at $37^{\circ} \mathrm{C}$ for 24 hours in darkened condition prior to imaging. For minimal medium with varying nutrient concentration, mid-exponential phase liquid cultures were washed three times with 1x M9 salts. They were then resuspended in M9 medium with appropriate amounts of glucose and ammonium chloride (for carbon and nitrogen variation, respectively). M9 agar substrates cast into the 3D-printed imaging chambers were inoculated at a concentration of $1 \times 10^{4} / \mathrm{mL}$, which ensured colonies were sufficiently spaced out and did not have to compete for nutrients with others in their proximity. It was also ensured that only one colony was visible in the field of view of the Mesolens at once, which prevented background signal from nearby colonies from reaching the detectors.

\section{E. coli growth characterisation under different nutrient conditions}

Mid-exponential growth phase liquid cultures of E. coli JM105-mini-Tn7-gfp were prepared as described above, then washed and resuspended in 1x M9 salts. The cultures were diluted to an $\mathrm{OD}_{600}$ of 0.04 , split in individual tubes and supplemented with appropriate amounts of the nutrient of interest. The nutrient concentrations were chosen to be between $0 \mathrm{mM}$ and $80 \mathrm{mM}$ - for comparison, the carbon and nitrogen concentrations in nominal M9 salts are $66.6 \mathrm{mM}$ and $18.7 \mathrm{mM}$, respectively. Aliquots $(200 \mu \mathrm{L})$ of liquid culture of each investigated concentration was added in triplicate to a black Nunc MicroWell 96-well optical-bottom plate with polymer base (ThermoFisher Scientific, US). Absorbance $\left(\mathrm{OD}_{600}\right)$ measurements were performed every 15 minutes for 24 hours, with the plate being held at $37^{\circ} \mathrm{C}$ and shaken continuously.

Growth curves were produced for each nutrient concentration thanks to the Gen5 microplate software (BioTek, USA), and exported to MATLAB (MathWorks, USA) for analysis. The average of the first absorbance value for each concentration was used as a baseline and subtracted from each growth curve. The y axis was displayed in logarithmic scale, in order to identify the exponential growth phase (the linear portion of the plot). A linear fit to this region was applied using MATLAB's Curve Fitting Toolbox by selecting an exponential function of the form $y=a \exp (b x)$, with the specific growth rate corresponding to the coefficient $b$. While error analysis was produced internally in MATLAB as R-squared, SSE and RMSE values, the error bars on the specific growth rate plots were calculated as the standard deviations across biological repeats for the same nutrient concentration. Note that the duration of the exponential growth condition varied depending on the nutrient concentration, and lasted between 6 and 18 hours, hence the data points corresponding to exponential growth were selected manually. Growth curves and specific growth rates were plotted in Python using matplotlib.

\section{Widefield epi-fluorescence and confocal laser scanning Mesolens imaging}

All the data in this work was acquired using the Mesolens, a custom-made optical microscope combining high numerical aperture (0.47) with low magnification $(4 \mathrm{x})$. These characteristics allow simultaneous 
imaging over a field of view of $6 \mathrm{~mm}$ x $6 \mathrm{~mm}$ with sub-cellular lateral resolution throughout $(700 \mathrm{~nm})$. Images can be acquired over a total thickness of $3 \mathrm{~mm}$ with an axial resolution of $7 \mu \mathrm{m}$. For widefield epi-fluorescence Mesolens imaging, GFP fluorescence was excited by a $490 \mathrm{~nm}$ pE-4000 LED (CoolLED, UK), and emitted fluorescent light was made to pass through a $540 \pm 10 \mathrm{~nm}$ bandpass filter before being detected by a VNP-29MC sensor-shifting CCD camera (Vieworks, South Korea). The camera uses sensorshifting to acquire 9 images for each pixel (each shifted by $1 / 3$ of a pixel width from the other, forming a square grid), effectively increasing the resolution to 259.5 MP [55]. In confocal laser scanning mode, GFP fluorescence was excited using a $488 \mathrm{~nm}$ laser (Multiline Laserbank, Cairn) at 5mW power. Fluorescence emission was filtered through a 525/39 nm bandpass filter (MF525-39, Thorlabs, USA) before being detected using a PMT (PMM02, Thorlabs, USA). The emission path also included a 505 nm longpass dichroic mirror (DMLP505R, Thorlabs, USA). Both widefield and confocal laser scanning microscopy were performed with the lens in water immersion, to match the refractive index of the mounting media (LB and M9), except the 0.5\% M9 agar datasets which were acquired with air immersion. This was needed to preserve biofilm structure, as colonies grown $0.5 \% \mathrm{M} 9$ agar were less adherent to the substrate and detached from it after addition of liquid mounting medium. The intra-colony channels formed by these biofilms remain evident even though the lower refractive index resulted in poorer spatial resolution (Figure 4d). The Mesolens collars were adjusted to minimise spherical aberrations.

\section{Image analysis}

Confocal z-stacks were displayed as a maximum intensity projection, colour coded by depth across the z-direction using the "Fire" lookup table from FIJI. Where necessary, median-filtered widefield epifluorescence Mesolens images (filter radius: 2 pixels) were deconvoluted using the proprietary Huygens Professional version 19.04 software (Scientific Volume Imaging, Netherlands). Deconvolution was performed after an in-built manual background subtraction and a theoretical point spread function estimation on Huygens, using the Classic Maximum Likelihood Estimation method with 50 iterations, a signal-to-noise ratio of 40 and a quality threshold of 0.01 .

To calculate colony base area, widefield Mesolens images were opened in FIJI, and thresholded using the "adjust threshold" tool, using the mean of gray levels as the threshold value. The "Wand (tracing) tool" was then used to select the biofilm mask, and the area was calculated using the "Measure" function. The base area measurements were systematically underestimated due to the thresholding method used by FIJI to create the binary mask. Nonetheless, by using the same thresholding method on all images we ensured that this limitation had comparable effects on all datasets. Biofilm thickness was calculated by plotting z-axis profiles of confocal z-stacks in FIJI and calculating the distance between the two minima on each profile.

Mesolens images were brightness- and contrast-adjusted using FIJI for presentation purposes [56].

\section{Intra-colony channel width calculation}

Intra-colony channel width was calculated using the image analysis workflow outlined in Figure 6 . The pipeline made use of the FIJI plugin "Polar transformer" [57], which performed an image transformation from polar to cartesian coordinates, as well as of two custom Python scripts. Initially, the coordinates of the centre of the colony were calculated using the "Measure" function on FIJI applied on an oval selection of the whole colony. The Polar Transformer plugin was then launched, and the origin of transformation was manually entered as the coordinates of the colony centre. The number of pixels for each degree in angular coordinates was set to 7200 (corresponding to 20 pixels for each degree). The polar-transformed image was contrast-adjusted using the "CLAHE" (Contrast Limited Adaptive Histogram Equalization) feature on FIJI with block size 60, maximum slope 3 and 256 histogram bins to facilitate channel identification. The image was then despeckled using the "Despeckle" function on FIJI in order to remove noise. The image was also inverted, making intra-colony channels appear light and cells dark, which facilitated the rest of the analysis. Vertical line selections were taken at different $\mathrm{x}$ positions on the polar-transformed image, corresponding to circumferences around the colony taken at different radial distances from its centre. Signal profiles were obtained for each line selection using the "Plot profile" feature on FIJI, and were exported to Python, where signal peaks were located thanks to a custom script using the "find_peaks()" function. For each dataset, peak thresholding was performed in order to exclude noise: peak prominence was chosen as $20 \%$ of the difference between maximum and minimum signal, and a minimum distance between adjacent peaks was selected as 9 pixels, which corresponds to the average length of an E. coli cell $(2 \mu \mathrm{m})$ on a Mesolens widefield epifluorescence image. 


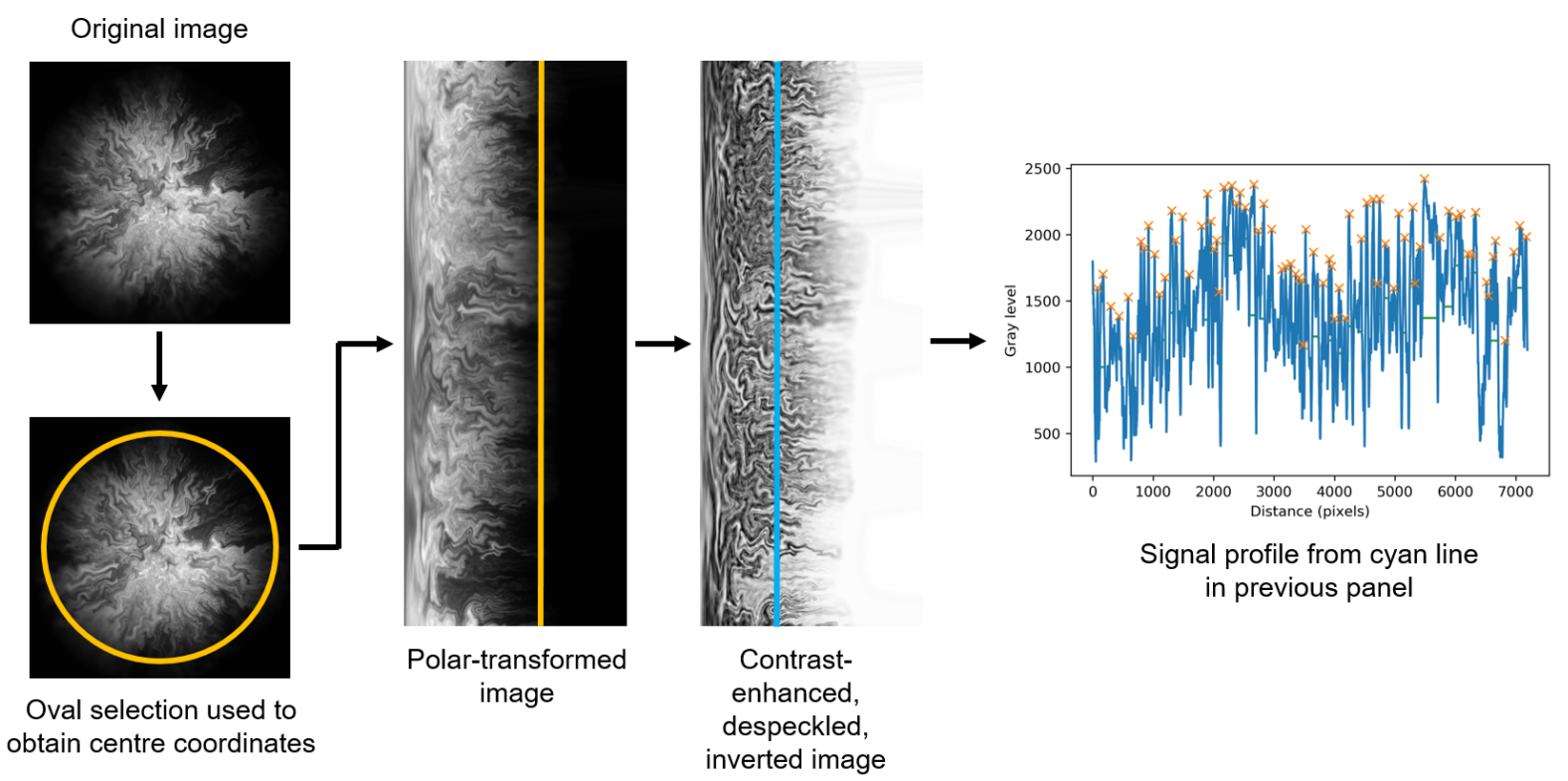

Figure 6: Image analysis workflow. A widefield Mesolens image is opened on FIJI, where an oval selection is used to obtain the coordinates of the centre of the biofilm. The coordinates are the input into the Polar Transformer FIJI plugin, which performs a transformation from polar to cartesian coordinates. The resulting image is locally contrast-enhanced, despeckled and inverted on FIJI. Signal profiles of vertical line selections are exported to Python, where the signal is analysed with a custom script in order to locate the peaks (orange crosses) and calculate the full-width at half-maximum (green horizontal lines) of each peak. The latter quantity corresponds to intra-colony channel width, converted from pixel units to $\mu \mathrm{m}$ using polar geometry.

Intra-colony channel widths were then calculated as the full-width at half-maximum of each peak. The actual width of the channels in $\mu \mathrm{m}$ was converted according to the radial distance from the centre, using polar coordinate geometry.

\section{Variation of intra-colony channel width along the radial dimension}

Intra-colony channel width was measured along whole circumferences at radius intervals of $50 \mu \mathrm{m}$, starting from an initial radius of $200 \mu \mathrm{m}$. This initial radius value was chosen because at lower values, polar transformed images were distorted and signal analysis was not reliable. Because of the ellipticity of the colonies, polar-transformed images appeared as rectangles with a non-straight right side (Figure 6), corresponding to the colony perimeter. Regions beyond which a full circumference could not be obtained for the colony were excluded from the analysis.

Channel widths were measured for colonies grown on the four nutrient conditions described in the Results section. Three biological replicates were used for each condition, giving a total sample size of 12 colonies. An exponential fit was applied to each plot of channel width against radius, and R-squared values for the fit were computed for each dataset. The number of channels identified by the script varied between radial positions, and was highest for the intermediate radii. This is likely due to the fractal nature of intra-colony channels, which have sharp changes in direction and fold on themselves inside the colony.

To compare channel widths across different nutrient concentrations of the substrate, intra-colony channel width was calculated at three normalised radial positions (maximum radius, mid-radius and $20 \%$ radius) to investigate the effect of nutrient concentration. Datasets were acquired for each of the following nutrient concentrations: $15 \mathrm{mM}$ carbon (8 colonies, giving 1062 channels), $200 \mathrm{mM}$ carbon (7 colonies, giving 1424 channels), $2.5 \mathrm{mM}$ nitrogen (9 colonies, giving 1524 channels) and $10 \mathrm{mM}$ nitrogen (4 colonies, giving 507 channels).

\section{Statistical analysis}

The comparison of colony base areas and channel widths under different nutrient availabilities and agar stiffness values was performed by means of a two-tailed t-test, assuming unequal variances between each 
pair of datasets. The test was performed using the Data Analysis package in Microsoft Excel 2016. Pvalues smaller than 0.05 were considered statistically significant. T-values and degrees of freedom were quoted for each t-test.

\section{Data and code availability}

The Python script used to calculate intra-colony channel width was written on Python version 3.7, and is deposited on GitHub (https://github.com/beatricebottura/biofilm_channel_width, DOI: 10.5281/zenodo.5786305). An example dataset to be analysed with the script is also available in the same repository. The remaining data used to generate channel width results is available upon reasonable request.

\section{References}

[1] Rodney M Donlan. Biofilms: microbial life on surfaces. Emerging infectious diseases, 8(9):881, 2002.

[2] Wen Yin, Yiting Wang, Lu Liu, and Jin He. Biofilms: the microbial "protective clothing" in extreme environments. International journal of molecular sciences, 20(14):3423, 2019.

[3] Zhizhong Yao, Rebecca M Davis, Roy Kishony, Daniel Kahne, and Natividad Ruiz. Regulation of cell size in response to nutrient availability by fatty acid biosynthesis in escherichia coli. Proceedings of the National Academy of Sciences, 109(38):E2561-E2568, 2012.

[4] Chenyi Fei, Sheng Mao, Jing Yan, Ricard Alert, Howard A Stone, Bonnie L Bassler, Ned S Wingreen, and Andrej Košmrlj. Nonuniform growth and surface friction determine bacterial biofilm morphology on soft substrates. Proceedings of the National Academy of Sciences, 117(14):7622-7632, 2020.

[5] Talaat E Shehata and Allen G Marr. Effect of nutrient concentration on the growth of escherichia coli. Journal of bacteriology, 107(1):210-216, 1971.

[6] Olga A Nev, Richard J Lindsay, Alys Jepson, Lisa Butt, Robert E Beardmore, and Ivana Gudelj. Predicting microbial growth dynamics in response to nutrient availability. PLoS computational biology, 17(3):e1008817, 2021.

[7] Mya R Warren, Hui Sun, Yue Yan, Jonas Cremer, Bo Li, and Terence Hwa. Spatiotemporal establishment of dense bacterial colonies growing on hard agar. Elife, 8:e41093, 2019.

[8] Fangchao Song, Megan E Brasch, Hao Wang, James H Henderson, Karin Sauer, and Dacheng Ren. How bacteria respond to material stiffness during attachment: a role of escherichia coli flagellar motility. ACS applied materials 8 interfaces, 9(27):22176-22184, 2017.

[9] Moritz Werb, Carolina Falcón García, Nina C Bach, Stefan Grumbein, Stephan A Sieber, Madeleine Opitz, and Oliver Lieleg. Surface topology affects wetting behavior of bacillus subtilis biofilms. npj Biofilms and Microbiomes, 3(1):1-10, 2017.

[10] Saida Benomar, David Ranava, María Luz Cárdenas, Eric Trably, Yan Rafrafi, Adrien Ducret, Jérôme Hamelin, Elisabeth Lojou, Jean-Philippe Steyer, and Marie-Thérèse Giudici-Orticoni. Nutritional stress induces exchange of cell material and energetic coupling between bacterial species. Nature communications, 6(1):1-10, 2015.

[11] Alice Cont, Tamara Rossy, Zainebe Al-Mayyah, and Alexandre Persat. Biofilms deform soft surfaces and disrupt epithelia. Elife, 9:e56533, 2020.

[12] James D Bryers. Medical biofilms. Biotechnology and bioengineering, 100(1):1-18, 2008.

[13] Thomas R Neu and John R Lawrence. Innovative techniques, sensors, and approaches for imaging biofilms at different scales. Trends in microbiology, 23(4):233-242, 2015.

[14] Oleg Paliy and Thusitha S Gunasekera. Growth of e. coli bl21 in minimal media with different gluconeogenic carbon sources and salt contents. Applied microbiology and biotechnology, 73(5):1169-1172, 2007.

[15] T Bauchop and SR Elsden. The growth of micro-organisms in relation to their energy supply. Microbiology, 23(3):457469, 1960.

[16] Nehir Kandemir, Waldemar Vollmer, Nicholas S Jakubovics, and Jinju Chen. Mechanical interactions between bacteria and hydrogels. Scientific reports, 8(1):1-11, 2018.

[17] Thomas A Witten and Leonard M Sander. Diffusion-limited aggregation. Physical review B, 27(9):5686, 1983.

[18] Hiroshi Fujikawa and Mitsugu Matsushita. Bacterial fractal growth in the concentration field of nutrient. Journal of the Physical Society of Japan, 60(1):88-94, 1991.

[19] Haluk Beyenal and Zbigniew Lewandowski. Combined effect of substrate concentration and flow velocity on effective diffusivity in biofilms. Water research, 34(2):528-538, 2000.

[20] Masayasu Mimura, Hideo Sakaguchi, and Mitsugu Matsushita. Reaction-diffusion modelling of bacterial colony patterns. Physica A: Statistical Mechanics and its Applications, 282(1-2):283-303, 2000.

[21] Rie Tokita, Takaki Katoh, Yusuke Maeda, Jun-ichi Wakita, Masaki Sano, Tohey Matsuyama, and Mitsugu Matsushita. Pattern formation of bacterial colonies by escherichia coli. Journal of the Physical Society of Japan, 78(7):074005074005, 2009.

[22] Eric Wolfsberg, Christopher P Long, and Maciek R Antoniewicz. Metabolism in dense microbial colonies: 13c metabolic flux analysis of e. coli grown on agar identifies two distinct cell populations with acetate cross-feeding. Metabolic engineering, 49:242-247, 2018. 
[23] Francisco Díaz-Pascual, Martin Lempp, Kazuki Nosho, Hannah Jeckel, Jeanyoung K Jo, Konstantin Neuhaus, Raimo Hartmann, Eric Jelli, Mads Frederik Hansen, Alexa Price-Whelan, et al. Spatial alanine metabolism determines local growth dynamics of escherichia coli colonies. Elife, 10:e70794, 2021.

[24] Fangchao Song and Dacheng Ren. Stiffness of cross-linked poly (dimethylsiloxane) affects bacterial adhesion and antibiotic susceptibility of attached cells. Langmuir, 30(34):10354-10362, 2014.

[25] Kristopher W Kolewe, Shelly R Peyton, and Jessica D Schiffman. Fewer bacteria adhere to softer hydrogels. ACS applied materials $\&$ interfaces, 7(35):19562-19569, 2015.

[26] Naresh Saha, Claire Monge, Virginie Dulong, Catherine Picart, and Karine Glinel. Influence of polyelectrolyte film stiffness on bacterial growth. Biomacromolecules, 14(2):520-528, 2013.

[27] Matthew AA Grant, Bartłomiej Wacław, Rosalind J Allen, and Pietro Cicuta. The role of mechanical forces in the planar-to-bulk transition in growing escherichia coli microcolonies. Journal of The Royal Society Interface, 11(97):20140400, 2014.

[28] Arturo A Massol-Deyá, Joanne Whallon, Robert F Hickey, and James M Tiedje. Channel structures in aerobic biofilms of fixed-film reactors treating contaminated groundwater. Appl. Environ. Microbiol., 61(2):769-777, 1995.

[29] Tim Tolker-Nielsen and Søren Molin. Spatial organization of microbial biofilm communities. Microbial ecology, 40(2):75-84, 2000.

[30] Alona Birjiniuk, Nicole Billings, Elizabeth Nance, Justin Hanes, Katharina Ribbeck, and Patrick S Doyle. Single particle tracking reveals spatial and dynamic organization of the escherichia coli biofilm matrix. New journal of physics, 16(8):085014, 2014

[31] Liam M Rooney, William B Amos, Paul A Hoskisson, and Gail McConnell. Intra-colony channels in e. coli function as a nutrient uptake system. The ISME journal, 14(10):2461-2473, 2020.

[32] Gail McConnell, Johanna Trägårdh, Rumelo Amor, John Dempster, Es Reid, and William Bradshaw Amos. A novel optical microscope for imaging large embryos and tissue volumes with sub-cellular resolution throughout. Elife, 5:e18659, 2016.

[33] Gang Chen and KA Strevett. Impact of carbon and nitrogen conditions on e. coli surface thermodynamics. Colloids and Surfaces B: Biointerfaces, 28(2-3):135-146, 2003.

[34] Jeremy M Chacón, Wolfram Möbius, and William R Harcombe. The spatial and metabolic basis of colony size variation. The ISME journal, 12(3):669-680, 2018.

[35] Julian WT Wimpenny. The growth and form of bacterial colonies. Microbiology, 114(2):483-486, 1979.

[36] Robert Waldrop, Alex McLaren, Francis Calara, and Ryan McLemore. Biofilm growth has a threshold response to glucose in vitro. Clinical Orthopaedics and Related Research R, 472(11):3305-3310, 2014.

[37] SJ Pirt. A kinetic study of the mode of growth of surface colonies of bacteria and fungi. Microbiology, 47(2):181-197, 1967.

[38] Lin Chen, Javad Noorbakhsh, Rhys M Adams, Joseph Samaniego-Evans, Germaine Agollah, Dmitry Nevozhay, Jennie Kuzdzal-Fick, Pankaj Mehta, and Gábor Balázsi. Two-dimensionality of yeast colony expansion accompanied by pattern formation. PLoS computational biology, 10(12):e1003979, 2014.

[39] G Reshes, S Vanounou, I Fishov, and M Feingold. Timing the start of division in e. coli: a single-cell study. Physical biology, 5(4):046001, 2008.

[40] Sarah Gingichashvili, Osnat Feuerstein, and Doron Steinberg. Topography and expansion patterns at the biofilm-agar interface in bacillus subtilis biofilms. Microorganisms, 9(1):84, 2021.

[41] Jing Yan, Chenyi Fei, Sheng Mao, Alexis Moreau, Ned S Wingreen, Andrej Košmrlj, Howard A Stone, and Bonnie L Bassler. Mechanical instability and interfacial energy drive biofilm morphogenesis. Elife, 8:e43920, 2019.

[42] Japinder Nijjer, Changhao Li, Qiuting Zhang, Haoran Lu, Sulin Zhang, and Jing Yan. Mechanical forces drive a reorientation cascade leading to biofilm self-patterning. Nature communications, 12(1):1-9, 2021.

[43] Boyang Qin, Chenyi Fei, Andrew A Bridges, Ameya A Mashruwala, Howard A Stone, Ned S Wingreen, and Bonnie L Bassler. Cell position fates and collective fountain flow in bacterial biofilms revealed by light-sheet microscopy. Science, 369(6499):71-77, 2020.

[44] Diego O Serra and Regine Hengge. Bacterial multicellularity: The biology of escherichia coli building large-scale biofilm communities. Annual review of microbiology, 75:269-290, 2021.

[45] Zhizhong Yao, Rebecca M Davis, Roy Kishony, Daniel Kahne, and Natividad Ruiz. Regulation of cell size in response to nutrient availability by fatty acid biosynthesis in escherichia coli. Proceedings of the National Academy of Sciences, 109(38):E2561-E2568, 2012.

[46] Jan-Ulrich Kreft, Cristian Picioreanu, Julian WT Wimpenny, and Mark CM van Loosdrecht. Individual-based modelling of biofilms. Microbiology, 147(11):2897-2912, 2001.

[47] Erik Alpkvist, Cristian Picioreanu, Mark CM van Loosdrecht, and Anders Heyden. Three-dimensional biofilm model with individual cells and continuum eps matrix. Biotechnology and bioengineering, 94(5):961-979, 2006.

[48] Laurent A Lardon, Brian V Merkey, Sónia Martins, Andreas Dötsch, Cristian Picioreanu, Jan-Ulrich Kreft, and Barth F Smets. idynomics: next-generation individual-based modelling of biofilms. Environmental microbiology, 13(9):2416-2434, 2011.

[49] Joao B Xavier, Cristian Picioreanu, and Mark CM Van Loosdrecht. A framework for multidimensional modelling of activity and structure of multispecies biofilms. Environmental microbiology, 7(8):1085-1103, 2005.

[50] Jing Yan, Andrew G Sharo, Howard A Stone, Ned S Wingreen, and Bonnie L Bassler. Vibrio cholerae biofilm growth program and architecture revealed by single-cell live imaging. Proceedings of the National Academy of Sciences, 113(36):E5337-E5343, 2016. 
[51] Raimo Hartmann, Praveen K Singh, Philip Pearce, Rachel Mok, Boya Song, Francisco Díaz-Pascual, Jörn Dunkel, and Knut Drescher. Emergence of three-dimensional order and structure in growing biofilms. Nature physics, 15(3):251$256,2019$.

[52] Knut Drescher, Jörn Dunkel, Carey D Nadell, Sven Van Teeffelen, Ivan Grnja, Ned S Wingreen, Howard A Stone, and Bonnie L Bassler. Architectural transitions in vibrio cholerae biofilms at single-cell resolution. Proceedings of the National Academy of Sciences, 113(14):E2066-E2072, 2016.

[53] Lotte Lambertsen, Claus Sternberg, and Søren Molin. Mini-tn7 transposons for site-specific tagging of bacteria with fluorescent proteins. Environmental microbiology, 6(7):726-732, 2004.

[54] Karen L Elbing and Roger Brent. Recipes and tools for culture of escherichia coli. Current protocols in molecular biology, 125(1):e83, 2019.

[55] Jan Schniete, Aimee Franssen, John Dempster, Trevor J Bushell, William Bradshaw Amos, and Gail McConnell. Fast optical sectioning for widefield fluorescence mesoscopy with the mesolens based on hilo microscopy. Scientific reports, $8(1): 1-10,2018$.

[56] Johannes Schindelin, Ignacio Arganda-Carreras, Erwin Frise, Verena Kaynig, Mark Longair, Tobias Pietzsch, Stephan Preibisch, Curtis Rueden, Stephan Saalfeld, Benjamin Schmid, et al. Fiji: an open-source platform for biological-image analysis. Nature methods, 9(7):676-682, 2012.

[57] Edwin Donnelly and Frederic Mothe. Polar transformer. https://imagej.nih.gov/ij/plugins/polar-transformer. html, 2007.

\section{Acknowledgements}

We thank Dr Ainsley Beaton (John Innes Centre, formerly University of Strathclyde) for the kind gift of the JM105-miniTn7-gfp strain. We also acknowledge Dr John Munnoch (University of Strathclyde), Dr Rebecca McHugh (University of Glasgow) and Dr Morgan Feeney (University of Strathclyde) for helpful discussions. Beatrice Bottura is supported by a University of Strathclyde Student Excellence Award. Gail McConnell is supported by the Medical Research Council [MR/K015583/1] and Biotechnology \& Biological Sciences Research Council [BB/P02565X/1, BBT011602]. Paul A. Hoskisson is supported by funding from BBSRC [BB/T001038/1 and BB/T004126/1] and the Royal Academy of Engineering Research Chair Scheme for long term personal research support [RCSRF2021 $11 \backslash 15]$.

\section{Author Contributions}

BB conducted all experiments and analysed all data. All authors were responsible for the experimental design. All authors prepared the manuscript.

\section{Competing interests}

The authors declare no competing interests. 
bioRxiv preprint doi: https://doi.org/10.1101/2021.12.17.473116; this version posted December 18, 2021. The copyright holder for this preprint (which was not certified by peer review) is the author/funder, who has granted bioRxiv a license to display the preprint in perpetuity. It is made available under aCC-BY-NC-ND 4.0 International license.

\section{Additional information}
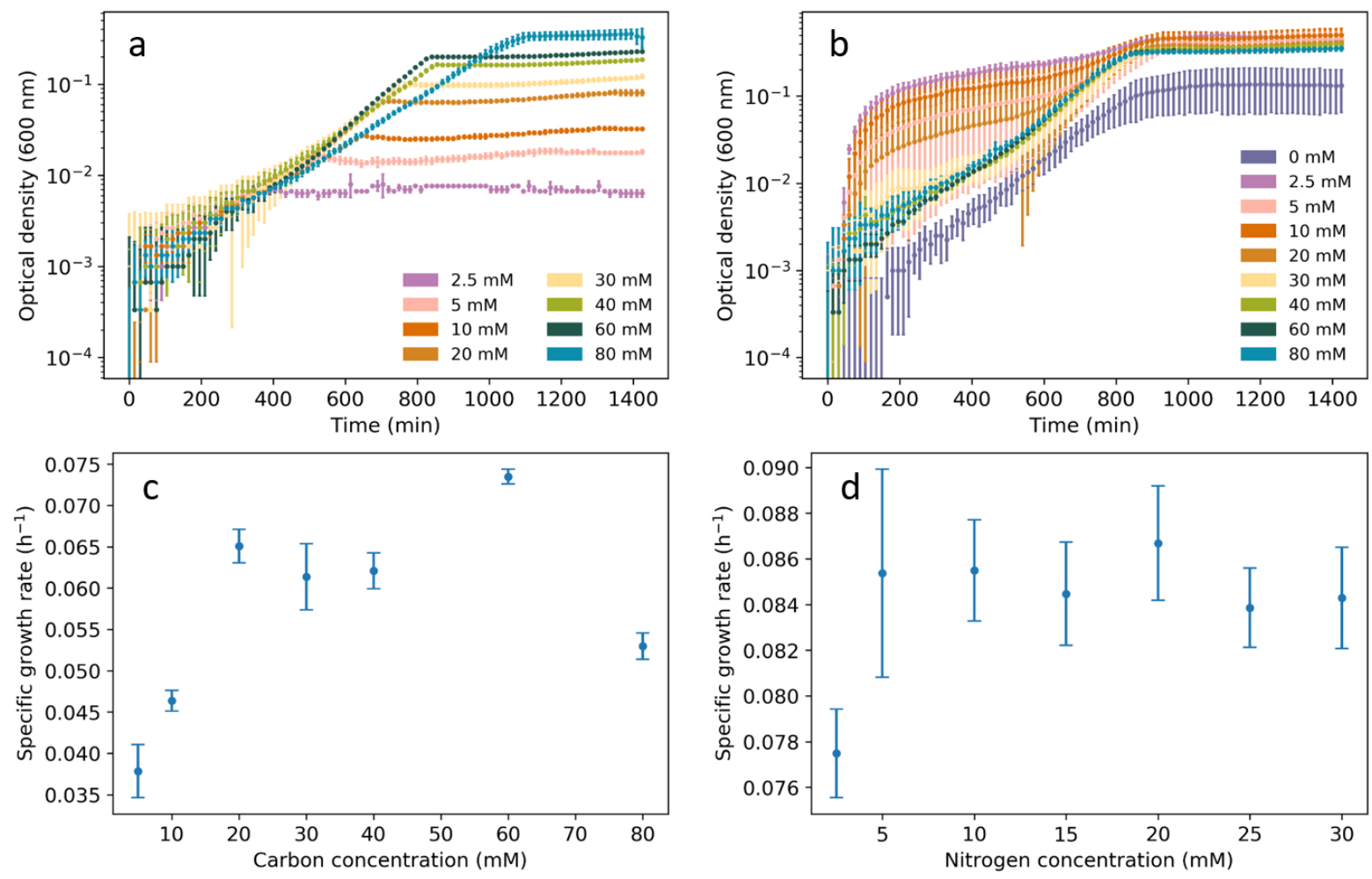

Supplementary Figure 1: Growth curves (top panels) and specific growth rates (bottom panels) of E. coli JM105mini-Tn7-gfp liquid cultures grown in M9 minimal medium with various carbon (a, c) and nitrogen (b, d) concentrations. Error bars on growth curve plots represent the standard deviation across three biological repeats. The growth curve for the lowest carbon concentration in a $(0 \mathrm{mM})$ is not shown as it consists of a baseline of non-growing cells. The data point at $80 \mathrm{mM}$ carbon (c) is likely due to saturation, especially if we consider that the nominal carbon concentration in $\mathrm{M} 9$ medium is $67 \mathrm{mM}$.

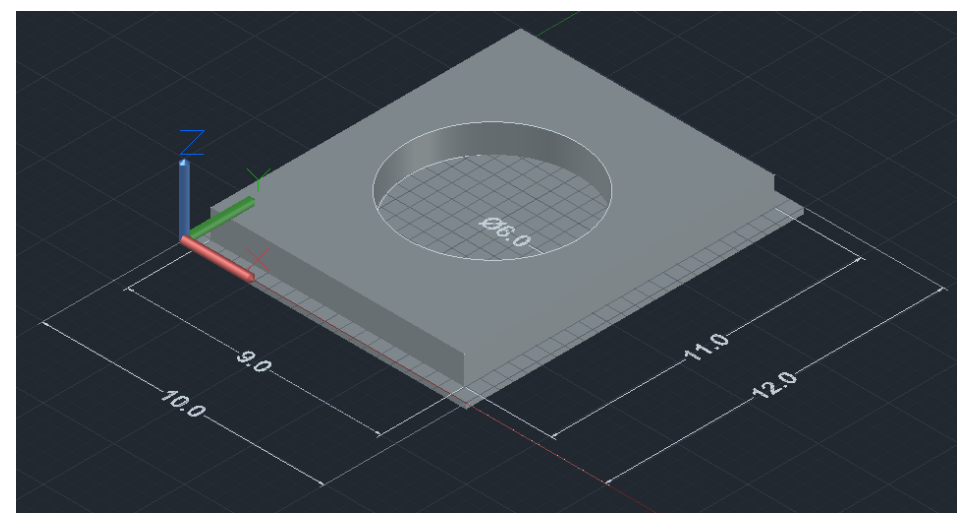

Supplementary Figure 2: 3D-printed imaging chamber used with the Mesolens. Measurements are given in $\mathrm{cm}$. 